\title{
Tangence
}

\section{Le sida, puisqu'il faut l'appeler par son nom...}

\section{Catherine Mavrikakis}

Numéro 42, décembre 1993

Le récit de soi

URI : https://id.erudit.org/iderudit/025794ar

DOI : https://doi.org/10.7202/025794ar

Aller au sommaire du numéro

Éditeur(s)

Tangence

ISSN

0226-9554 (imprimé)

1710-0305 (numérique)

Découvrir la revue

Citer cet article

Mavrikakis, C. (1993). Le sida, puisqu'il faut l'appeler par son nom... Tangence, (42), 146-159. https://doi.org/10.7202/025794ar d'utilisation que vous pouvez consulter en ligne.

https://apropos.erudit.org/fr/usagers/politique-dutilisation/ 


\section{Le sida, puisqu'il faut l'appeler par son nom... Catherine Mavrikakis}

Peut-on desceller la bouche pour en faire le lieu de l'autobiographie? Et peut-on affirmer que le récit de soi se veut la démolition d'une bouche-caveau où le sujet se serait avalé, lové, enterré tout entier? Ce sont ces interrogations que posent les écrits d'Hervé Guibert sur ce qu'il est convenu d'appeler "son" sida. Hervé Guibert a toujours écrit, mais l'annonce de sa maladie précipite l'élaboration d'une série de textes autobiographiques qui relatent la vie de l'auteur et du même coup la vie du sida en celui-ci. Écrire sur soi, c'est aussi écrire sur la maladie, dans, pour et contre elle, et ériger, somme toute, le sida en oeuvre d'art et même en signature. Que reste-t-il du nom de l'auteur dans cette fortification du nom du sida? À quel processus de deuil le sujet et son nom sont-ils contraints dans l'imminence de la mort?

Cytomégalovirus ${ }^{1}$, tel est le nom d'un des derniers ouvrages de Guibert. Le titre du livre devient le nom de la maladie, un cryptogramme à l'usage de ceux qui savent ou qui veulent savoir, une inscription à déchiffrer. De même Le protocole compassion$n e l$, écrit un an avant la mort de son auteur, fait référence au cérémonial thérapeutique et médical et ne s'entend que dans son écho au mal. C'est bel et bien le nom de la maladie qui se grave aux côtés du nom de l'auteur sur la page de couverture. Et tous les textes autobiographiques de la fin de la vie porteront en eux signes, sigles, abréviations et acronymes (AZT, DDI, DHPJ, HIV, T4, FDA, HAD, PL, IRM, T.B., LAV, AFP, TGV) qui viennent rappeler le mot originel, S.I.D.A. impossible parfois à prononcer ${ }^{2}$. Cette contagion de l'abréviation que subit le langage se manifeste dans une stratégie du secret. Le sigle cache l'innommable, le nom de la mort et fait de celle-ci un nom propre bardé de majuscules. Les noms des personnages, eux aussi, souffrent de la maladie de l'abréviation (V.,Z.,C., T.,M.,D.,B., etc.) ou sont des pseudonymes

1 Hervé Guibert, Cytomégalovirus, Paris, Seuil, 1992.

2 Guibert écrit à propos du nom de sa maladie: "Voyez comme j'ai du mal de nouveau à prononcer le mot ", Hervé Guibert, Le protocole compassionnel, Paris, Gallimard, 1991, p. 17. 
de noms connus (Foucault alias Muzil, Adjani alias Marine) ${ }^{3}$. Cacher, travestir le nom en le codifiant, c'est toujours effacer la vérité du nom de la maladie, tout en ne cessant jamais de l'inscrire sous un autre nom encodé, donc déchiffrable. "Le sida [...] aura été pour moi un paradigme dans mon projet du dévoilement de soi et de l'indicible. " ${ }^{4}$ En fait, chez Guibert la stratégie du secret, du cryptogramme, du S.I.D.A, est avant tout une tactique pour dire ce qui ne peut être appelé par son nom de mort. Tout dire pour dire le sida sous un autre nom, pour effacer son sens premier, c'est à cette exigence que le récit répond. Trahir tous les secrets de tous et même les siens fait partie du processus scripturaire. À condition, bien sûr, de trahir encore, de trahir le nom de la maladie et tous les noms des amis-personnages. Dans cette partie de cache-cache avec le nom de la mort, l'auteur de l'autobiographie brouille les pistes, nomme dans le parasitage le plus total. Le sida, comme abréviation et comme code, est disséminé à travers le récit sur soi. Il est la matière de l'œuvre en tant qu'inscription jamais fixée de la mort. Il ne reste plus alors qu'à écrire sans cesse, jusqu'à la mort et dans l'ajournement de celle-ci, pour faire advenir tous les noms du sida et pour en découvrir un sens caché, dans une nouvelle abréviation, qui ne serait pas mortifère. Le DDI, par exemple, nom d'un médicament contre le sida, pourrait devenir le sigle capable de transformer la signification de toute abréviation. Guibert, telle une Shéhérazade du sida, endort celui-ci en le rebaptisant sans fin pour l'empêcher de se nommer pour de bon. Ce travail sur l'abréviation et la mort n'est pas sans rappeler ce que dit Michel de M'Uzan sur l'angoisse de mort chez Tolstoï exprimée dans son Joumal, écrit autobiographique:

Après la dernière notation de la journée, Tolstoï avait coutume d'inscrire la date du lendemain, en ajoutant toutefois un "si je vis encore" dicté sans doute par une crainte superstitieuse. Par la suite, désireux de gagner du temps ou de ne pas trop défier le sort, il réduisit les quatre mots à leurs initiales, ce qui en français eût donné s.j.j.e.e. ${ }^{5}$

Ce signe codé, s.j.v.e., qui vient témoigner de l'angoisse de castration et reproduire, dans sa coupure d'une phrase complète, la

3 Guibert nous dit qu'“évidemment tous les noms ont été changés "; dans Hervé Guibert, op. cit., p. 160.

4 Hervé Guibert, op. cit., p. 247.

5 Michel de M'Uzan, De l'art à la mort, Paris, Gallimard, 1977, p. 152. 
coupure qu'inflige la mort, est aussi l'indice manifeste de ce que M'uzan appelle "le travail du trépas "6. Tout homme, et particulièrement le malade condamné, doit faire le deuil de soi. Dans ce "juste avant la mort" le sujet connaît un élan pulsionnel et un renouveau d'impulsion créatrice, tel Guibert qui, à la fin de ses jours, se met à vivre en ne cessant d'écrire ou de tourner des films. Mais cette énergie n'existe que dans la possibilité de l'énoncé de sa mort prochaine, que dans l'abréviation mais aussi dans le déni partiel de la mort. Par l'abréviation, le sujet émet l'hypothèse de sa mort, mais tente de conjurer le sort par cette formule magique dont le sens est incertain. La formule codée fonctionne à deux niveaux, elle prépare à la mort, mais elle tente de déjouer celle-ci et d'inventer continuellement un nouveau sens à l'énoncé fatal. Trahir avant tout le nom de la mort, voilà le rôle de l'écrit guibertien.

Qu'advient-il du nom d'Hervé Guibert à l'intérieur de cette trahison de tous les noms?

Il y a deux jours, le 9 janvier, mes parents m'ont téléphoné hier pour m'en prévenir, est né le fils de ma sœur, qu'elle a décidé d'appeler Hervé, ignorant tout de ma maladie et de ma fin voisine probable, mais les pressentant peut-être, voulant me faire la surprise au dernier moment, me l'annonçant au déjeuner de Noël auprès de notre grand-tante Louise, alors que je venais de faire manger à l'hôpital notre autre grand-tante Suzanne, ajoutant qu'elle avait eu la bonne idée supplémentaire d'appeler son fils Hervé Guibert puisqu'elle avait récupéré son nom de jeune fille et que le nouveau père ne tenait pas spécialement à donner son nom à cet enfant, et ma sœur me disait tout cela à moi, qui avais toujours pensé qu'elle était une personne parfaitement équilibrée. ${ }^{7}$

Le nom de l'auteur ici devient le nom de l'autre, de la descendance indirecte. Le baptême par la sœur est signe, signe de mort pour le frère et le nom subit un effacement au profit d'autrui. De même, un autre personnage de Cytomégalovirus s'appelle H. G., portant ainsi les initiales de l'auteur. Il y a une multiplication des Hervé et des Hervé Guibert et la nomination semble toujours être liée à la proximité de la mort. Cette prolifération de son nom

$6 \quad$ Ibid., p. 161.

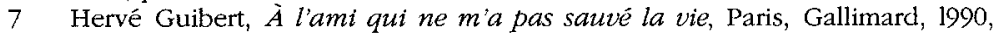
p. $69-70$. 
peut vouloir signifier un legs possible du nom et ainsi pourrait insérer le narrateur dans une lignée où le nom persiste comme reproduction, double. Aucune altération du nom n'aura lieu; à Hervé Guibert survit Hervé Guibert, sans même que le prénom du descendant comporte une marque de distinction comme l'ajout de junior le permet. Mais il semble, au contraire, que cette répétition du nom cache le nom comme abréviation, sida, cryptogramme à déchiffrer. Dans un texte truffé de sigles, comment ne pas entendre l'abréviation R. V. dans Hervé (qui d'ailleurs fait écho à celle de rétrovirus). Le nom lui-même est en position de sida et est peutêtre répété dans les écrits autobiographiques comme cryptogramme dont le sens est à redécouvrir. Cet appel à l'aide, cette demande d'interprétation du nom ou de la lettre n'est pas inconsciente chez Guibert. Elle relève plutôt d'une volonté de laisser le sens à autrui, de ne pas savoir l'issue et la signification de toutes choses. Ainsi, on peut lire ces lignes pour le moins étranges:

Depuis tout à l'heure, je recherche le nom de la ville où l'avion a fait escale avant de se poser à Casablanca, j'ai l'impression que ça commence par un $d$, c'est une des villes les plus connues du Maroc, sur la Côte Atlantique, au sud de Tanger, mais je ne le retrouve pas. Ce n'est pas Djerba, bien sûr, qui me vient pourtant à l'esprit, mais si le $\mathrm{D}$ était une fausse piste? ${ }^{8}$

Le narrateur ne cherche pas sur une carte la solution à ses questions sur le sens de l'initiale ou de la lettre. Au lecteur, de se rappeler le nom de cette ville, de fouiller les atlas ou encore de faire appel à sa mémoire. Ce passage est une véritable invitation au décodage tout en étant une mise en garde contre la lettre D qui peut conduire à une mauvaise interprétation. Le narrataire est convié à rétablir le sens mais aussi à se méfier des inscriptions de Guibert. Ce qui s'énonce ici, c'est un "déchiffrez-moi, malgré moi", car le narrateur ne veut plus du sens qu'il pourrait donner aux abréviations et au sida, bien évidemment.

Et pourtant, le sida, comme abréviation continue son ouvre et contamine tous les textes, si bien que Cytomégalovirus, le dernier écrit sur soi, est un récit perpétuellement coupé, au style très télégraphique, rempli de mots tronqués (réhos: réhospitalisation, ophtalmo, mégalo, porte-à-cath). Cette fragmentation du 
langage, cette syncope infligée aux mots et aux phrases est basée sur l'idée d'une dévoration de la langue. Ce processus avait commencé dès Á l'ami qui ne m'a pas sauvé la vie, lorsque Guibert comparait l'aggravation du virus dans le corps à la progression du style de Thomas Bernhard dans les écrits du narrateur:

[...] parallèlement donc au virus HIV la métastase bernhardienne s'est propagée à la vitesse grand $\mathrm{V}$ dans mes tissus et mes réflexes vitaux d'écriture, elle la phagocyte, elle l'absorbe, la captive, en détruit tout naturel et toute personnalité pour étendre sur elle sa domination ravageuse. ${ }^{9}$

L'écriture de Guibert se trouve dévorée, phagocytée par celle de Bernhard. Or, cette dévoration va jusqu'au grignotement du langage dont il ne restera plus que des lettres, des morceaux tronqués dans les écrits suivants. L'écriture se trouve mangée tout comme le corps:

L'homme mange des animaux, des agneaux, des cochons de lait, des entrailles, des cervelles, des reins et des rognons blancs, des cœurs, des poulpes, des batraciens frits, des organismes palpitants, des huîtres crues. Le sida microscopique et virulent, mange l'homme, ce géant. ${ }^{10}$

La métaphore de la voracité fait du sida le maître absolu. Ailleurs ${ }^{11}$, Guibert compare le sida au jeu du Pacman qui bouffe tout sur son passage. Tout comme Thomas Bernhard, le sida gagne car il sait avaler, et avaler en l'homme tout ce que celui-ci a absorbé. Il est la dévoration par excellence et sa présence s'accompagne tout au long de l'autobiographie d'une perte d'appétit chez le narrateur qui souffre d'anorexie mentale ${ }^{12}$ et de problème de déglutition ${ }^{13}$. Se priver de manger, c'est affamer la maladie, mais c'est aussi s'affamer. Les atteintes contre le virus sont en même temps des attaques contre soi et tout médicament est poison:

[...] je n'ai toujours pas avalé la moindre gélule d'AZT. Sur la notice du médicament, chaque malade peut lire la liste des troubles plus ou moins gênants qu'il peut entraîner:

"nausées, vomissements, pertes d'appétit, maux de tête, éruption cutanée, douleurs du ventre, douleurs musculaires, fourmillement

9 Hervé Guibert, Á l'ami qui ne m'a pas sauvé la vie, p. 216.

10 Ibid., p. 158.

11 Hervé Guibert, À l'ami qui ne m'a pas sauvé la vie, p. 13.

12 Hervé Guibert, Le protocole compassionnel, p. 226.

13 Ibid., p. 11. 
des extrémités, insomnies, sensation de grande fatigue, malaise, somnolence, diarrhée, vertiges, sueur, essoufflement, digestion difficile, trouble du goût, douleurs thoraciques, toux, baisse de la vivacité intellectuelle, anxiété, besoin fréquent d'uriner, dépression, douleurs généralisées, urticaire, démangeaisons, syndrome pseudogrippal. " Désactivation de l'appareil génital, désintégration des facultés sensuelles, impuissance. ${ }^{14}$

La liste est longue et montre à quel point le corps fait corps avec le virus de telle sorte qu'il est impossible d'envisager la mort de l'un sans celle de l'autre. Guibert se doit d'avaler les médicaments pour que le sida, qui avale l'homme, ingurgite à son tour le poison. Mais dans cet engloutissement des bouches, le narrateur ne peut plus manger, seul le sida est avide.

C'est par la bouche pourtant, qui est pendant la progression de la maladie de plus en plus frappée d'interdit, que le sida s'est déclaré et c'est là qu'il réside. Un "abcès monstrueux" ${ }^{15}$ apparu en 1983 montre l'installation du mal. Le mode de manifestation de celui-ci n'est pas sans raviver dans l'esprit du narrateur le souvenir du mode de transmission du virus. Guibert se souvient d'une sortie effectuée dans une boîte homosexuelle de Mexico et écrit:

La vieille pute avait franchi le pas que j'avais refusé à l'actrice italienne, elle avait sans prévenir fourré sa langue au fond de ma gorge, et, de milliers de kilomètres plus loin, son baiser revenait à chaque sensation de douleur que produisait mon abcès pour le creuser plus profondément, comme la pointe d'un fer chauffé à blanc. ${ }^{16}$

C'est donc la bouche qui est le lieu de la maladie et refuser, par l'anorexie, la nourriture revient à refuser de nouvelles entrées au sida pour ne laisser l'accès qu'aux médicaments. Dans Le protocole compassionnel, Guibert décrit le traumatisme de la fibroscopie qui consiste à faire entrer un tuyau dans la bouche qui s'enfoncera jusqu'à l'estomac. Ne rien faire entrer dans la bouche occupe les pensées du narrateur et les deux tuyaux, l'œsophage et la trachée, sont des chemins de la mort, car très vite la respiration va être menacée:

Je réalisai alors corporellement avec le passage du liquide qui m'étouffait puis celui du long tuyau noir flexible, cette évidence 
152

que nous avons deux sortes de tuyaux, au niveau de la gorge, l'un qui mène au ventre à la nourriture, à la chiasse et aux ulcères comme à la monstrueuse fibroscopie, l'autre par lequel mon père m'avait fait rendre la pastille jaune interdite qui m'étouffait, en me secouant les pieds. C'était par ce tuyau-là que l'eau de mer envahissait les poumons des noyés [...]. Ce tuyau-là menait à la mort assurément, il menait à la cause de la mort pour la plupart des malades du sida, la mort par suffocation. ${ }^{17}$

L'orifice buccal se constitue comme passage pour la mort, mais il devient aussi un lieu clos, hermétique dans lequel le narrateur va s'enfermer, ne laissant plus rien entrer ni sortir. Cet enfermement de l'oral qui va se trouver tout à fait interdit d'existence se produit à l'occasion de la réitération d'un souvenir d'enfance: "le retour de la pastille jaune et mortelle que le père a su arracher aux tuyaux de la mort". Le père a déjà manifesté son contrôle sur la bouche de son fils, il a su reculer la mort de l'enfant en mettant Hervé à l'envers, (en le secouant par les pieds), en faisant de l'orifice oral un lieu anal, par le renversement du corps, et ainsi en prêtant à la bouche une maîtrise sadique-anale. Ce n'est pas la première fois que le père intervient dans la bouche du fils. Il semble au contraire avoir particulièrement la haute main sur l'intérieur de son fils. Déjà dans Mes parents, récit qui ne fait pas partie des textes du sida, mais qui est néanmoins autobiographique, Hervé Guibert racontait une scène de bouche:

Ma mère vient me chercher à mon tour, elle m'emmène devant la porte de la cuisine où mon père m'agrippe, me prend les bras, les passe dans les manches d'une blouse à l'envers qu'il se met à lier, lui-même est en blouse blanche et ma mère en enfile une autre, il y a là une horrible odeur d'éther qui glace méchamment la peau, mon père tient une seringue dans sa main, ma mère me serre contre elle et tente de bander d'une main mes yeux affolés et de bâillonner de l'autre mes hurlements tandis que mon père me fait la piqûre. Puis on me monte en larmes dans notre chambre où je retrouve ma sœur, elle aussi piquée et furieuse. Mes parents avaient trouvé préférable, ou plus économique, ce petit drame, ce coup fourré, ce mensonge, ce stratagème compliqué à une intervention usuelle dans un cabinet médical. ${ }^{18}$

17 Hervé Guibert, Le protocole compassionnel, p. 77-78.

18 Hervé Guibert, Mes parents, Paris, Gallimard, 1986. 
La scène qui a lieu est remarquable par son dit et son non-dit. Elle laisse interdits les mots qui feraient référence à l'évocation d'une opération, qui est ici l'extraction des amygdales de la bouche du narrateur et de la sœur de celui-ci. La mise en silence, dans l'écriture, de l'événement qui a traumatisé montre le pouvoir mortifère et stupéfiant du père sur la bouche, la gorge et tout ce qui rappelle l'oral de l'enfant. La langue ne peut plus nommer ce qu'elle a vécu et les mots sont prisonniers dans la bouche, innommables. Le narrateur raconte l'outrage sur le corps en le circonscrivant; sa parole tourne autour de ce qui n'a pas de mot, comme si même sur cela seul le père avait le pouvoir de parler. Ce qui est revécu plus tard quand le sida attaque précisément les tuyaux, ce n'est pas tant la menace que constitue le père sadique, mais plutôt la toute-puissance du père tout aussi bénéfique que maléfique. Le père peut faire rendre la pastille jaune et sauver le fils de la mort. C'est pour cela que Guibert malade, alors qu'il n'a plus la force de nager et craint la noyade qu'il a déjà comparée à la mort des sidéens, pense à son père et le voit comme un sauveur:

[...] je ne peux plus faire trois brasses de suite, j'ai des moignons, je suis à quelques pas du bord de l'eau, mais je me noie. J'ai besoin de m'agripper aux hanches de mon père pour qu'il me ramène à la plage. ${ }^{19}$

Le père semble vraiment être celui qui a contrôle sur les tuyaux du fils et qui peut le sauver de la noyade propre à tous les sidéens. Or, le processus de deuil de soi, ce travail du trépas, tel que nous l'avons vu défini par Michel de M'Uzan, ne peut avoir lieu que par introjection. Le moment de cette introjection qui correspond à un processus d'élargissement du moi, ici capable de se saisir comme mort, s'élabore la bouche vide. Comme le disent Nicolas Abraham et Maria Torok, "introjecter un désir, une douleur, une situation, c'est les faire passer par le langage dans une communion de bouches vides" ${ }^{20}$. En effet, le vide de la bouche chez le nourrisson qui subit l'absence du sein maternel est comblé par l'auto-remplissement phonatoire, par la conversion en mots durant le développement ultérieur de l'enfant. Le deuil de la mère se fait dans la bouche vide commune à tous les sujets

19 Hervé Guibert, Le protocole compassionnel, p. 149-150.

20 Nicolas Abraham et Maria Torok, L'écorce et le noyau, Paris, Aubier-Flammarion, 1978 , p. 263. 
parlants. L'introjection de sa propre absence possible ne peut se faire que dans l'opération d'auto-appréhension de son absence inscrite dans les mots. Or, la bouche de Guibert ne lui appartient pas. Elle est totalement envahie par la maladie et toute maîtrise sur elle appartient à la figure paternelle. L'absence de soi, le deuil possible du moi ne peut venir grossir le moi car tout l'oral est envahi par l'oral du sida (n'oublions pas que le sida est une bouche dévorant la bouche de Guibert) et par la pulsion sadiqueanale que le père a gardé pour lui. En effet, quand le père du petit Hervé a fait subir à son fils cette castration innommable qui consiste (car nous voulons la nommer) à l'ablation des amygdales, Hervé ne s'est ni approprié par le langage le vide que le père a créé dans la bouche, ni la pulsion sadique du père, ni la mort d'une image idéale du père. En ne mettant pas totalement en mots, en bouche, ce vide et cette pulsion, Hervé Guibert n'introjecte rien. Le récit autobiographique sur l'opération tente pourtant de dire l'innommé, mais en même temps de le garder secret. Au lecteur de combler le vide, de parler pour Guibert, de lui faire introjecter son manque. Mais la maladie, le sida, va réactiver ce qui avait débuté dans Mes parents, par le récit autour de l'opération: le besoin d'introjection. L'innommé qui est resté à travers la gorge d'Hervé demande à venir à la parole, d'où une somatisation massive dans le lieu de l'oral et une localisation constante de la maladie dans les tuyaux. Guibert doit amorcer le deuil de lui et lorsqu'il n'est pas dépressif et anorexique, il se met à faire revivre sa bouche:

Je manque tellement de chair sur mes propres os, dans mon ventre, puisque je ne mange plus ni viande ni poisson depuis des mois, sur ma langue et sous mes doigts, dans mon cul et dans ma bouche ce vide que je n'ai plus envie de combler, que je deviendrais volontiers cannibale. Quand je vois le beau corps dénudé, charnu d'un ouvrier sur un chantier, je n'aurais pas seulement envie de lécher, mais de mordre, de bouffer, de croquer, de mastiquer, d'avaler. Je ne découperais pas à la mode japonaise un de ces ouvriers pour le tasser dans mon congélateur, je voudrais la chair crue et vibrante, chaude, douce et infecte. ${ }^{21}$

[...] j'ai saisi le verre d'eau posé devant moi, et j'ai admiré sa finesse par transparence, $j$ 'ai fait remarquer à quel point il était

21 Hervê Guibert, Le protocole compassionnel, p. 90. 
fin, et à ce moment-là mon corps, mes réflexes, mes dents ont accompli quelque chose que ma pensée n'avait pas décidé et à quoi elle n'avait même pas songé: au lieu de boire l'eau comme je m’apprêtais à le faire, j'ai croqué le verre, et je me suis retrouvé la bouche pleine de dizaines et de dizaines de petits morceaux de verre, dont je ne savais pas s'ils avaient blessé ou non ma langue, je n'avais aucun goût de sang dans la bouche. C'est quand j'en ai retiré les morceaux un par un, sans rien dire, comme au ralenti, que j'ai compris pourquoi mon corps avait fait ce geste malgré moi, c'était un geste de protestation, j'ai dit à Hector: "Comme ca vous pouvez dire à cette bonne femme que j'ai les dents encore plus longues qu'elle ne l'imaginait, que je bouffe même le verre ${ }^{*}{ }^{22}$

Guibert montre dans ses écrits une voracité égale à celle du sida.

L'acte manqué, que Guibert analyse et qui consiste à s'emplir la bouche de morceaux de verre pour montrer l'envie de dévorer, qui s'empare du narrateur, aurait pu conduire celui-ci à un accident grave. Au contraire cet acte manqué met en scène la possibilité de la mort, mais surtout le contrôle sur ce qui menace le sujet. On peut dire qu'en bouffant du verre le sujet s'approprie les pulsions sadiques du père, se les octroie dans la bouche. Guibert se donne une maîtrise sadique-orale. Cette absorption de la pulsion, cette intégration d'un contrôle oral se manifeste dans un cannibalisme presque réalisé dans la dévoration machinale du verre. Si Guibert retire les morceaux de verre de sa bouche "sans rien dire", il va prendre la parole, après avoir vidé sa bouche et symboliser son acte. Ce qui a lieu dans ce passage c'est une mesure d'anti-incorporation ${ }^{23}$ telle que celle que Torok et Abraham repèrent dans le cannibalisme:

La nécrophagie, toujours collective, se distingue également de l'incorporation; toute fantasmatique qu'elle soit à l'origine, sa réalisation en groupe en fait un langage - en mettant en scene le fantasme d'incorporation - à la fois que l'introjection de la perte est impossible et qu'elle a déjà eu lieu. Elle a pour effet d'exorciser le penchant, qui pourrait naître avec le décès, d'une incorporation psychique. Elle serait donc, non pas une

22 Ibid., p. 129.

23 L'incorporation est une introjection impossible qui introduit une chose imaginaire dans la bouche puisque les mots ne sont pas venus combler le vide du sujet. Elle a pour effet de supprimer illusoirement l'idée d'une lacune à combler à l'aide mots. 
156

variété d'incorporation, mais une mesure préventive d'antiincorporation. ${ }^{24}$

Guibert ici par sa mise en bouche du verre et par la mise en mots de cet acte fait précisément une tentative pour ne pas garder dans le silence et dans la bouche la mort possible de lui-même. Il résiste à l'incorporation. Si, lors de l'ablation des amygdales, il n'avait pu mettre de mots sur ce qui lui était arrivé, s'il avait gardé quelque chose de l'image paternelle intacte, s'il avait laissé à autrui la maîtrise sur l'oral, cette fois-ci son acte manqué lui permet de réussir à ne pas garder intacte la mort d'une image aimée. Au contraire. Guibert absorbe la mort possible, le verre, et la retire de sa bouche comme le père avait retiré la pastille. Ce faisant, le narrateur s'approprie sa bouche, et par la parole à autrui, s'approprie sa mort. "J'ai les dents longues et même la mort, je serai capable de l'avaler. "Mais ce n'est qu'une capacité devenue langage et Guibert n'a pas besoin de mourir tout de suite pour prouver ses aptitudes à avaler la mort. Celle-ci n'a pas besoin de lui rester en travers de la gorge ou de lui arracher les tuyaux. La mort est retirée et les mots dans la bouche se l'approprient sans faire du mal. Ainsi l'anti-incorporation cannibalique se transforme en introjection, en mise en mots, mais il aura fallu en passer par cette menace de l'incorporation, de la mort dans la gorge et par l'échec de celle-ci dans l'anti-incorporation. Et tout ce processus est directement lié à l'introjection ratée de l'enfance durant l'épisode des amygdales.

Lorsque Guibert parle de son désir cannibalique pour un ouvrier, dans le passage qui a déjà été cité, la bouche est mentionnée comme vide que le narrateur n'a plus envie de combler. Et c'est dans ce non-désir de remplir qu'apparaît l'envie de manger autrui. On voit ainsi que le cannibalisme n'est pas là pour boucher la bouche, mais pour marquer le vide, pour le célébrer par les mots. En ce sens, il est vraiment chemin vers l'introjection. Mais Guibert risque toujours de ne pas pouvoir sortir de la prison-bouche et d'enterrer vivants les mots qui bloquent sa gorge. La commémoration de l'incorporation devenue antiincorporation menace le sujet d'un grand enfermement. Un autre acte manqué vient révéler quelque chose de ce risque:

À peine suis-je entré dans ma cave pour récupérer en un coup de vent mon vieil aspirateur, que la porte blindée, que j'avais

24 Nicolas Abraham et Maria Torok, op. cit., p. 265. 
ouverte avec le trousseau de trois clés identiques, la rabattant largement contre la paroi et laissant imprudemment le trousseau à l'extérieur de la porte, sans aucun courant d'air, comme si une main invisible l'avait poussée dans mon dos, se referme sur moi. Plus moyen de sortir. ${ }^{25}$

Alors je me vis réellement, découvert des mois après, crevé dans cette cave, de soif, de faim, de froid et d'épuisement nerveux comme les écoliers de la Villa Médicis, squelette recroquevillé sous des cartons. J'avais un stylo dans ma poche, et des morceaux de papier, je pouvais au moins écrire, écrire mes derniers mots. ${ }^{26}$

Guibert, atteint du sida, a failli mourir enterré vivant, dans cette cave où il s'est enfermé par mégarde avec du papier et un stylo... L'écriture s'accommoderait de cet ensevelissement et de ce dessèchement du corps. Mais là encore, l'expulsion de la pastille aura lieu et Guibert sortira de son emprisonnement. La mise en scène d'une mise au tombeau et la résurrection qui en résulte n'est pas sans faire penser à un souvenir d'enfance et aux premiers pas dans l'écriture:

Depuis que j'ai douze ans, et depuis qu'elle est une terreur, la mort est une marotte. J'en ignorais l'existence jusqu'à ce qu'un camarade de classe, le petit Bonnecarrère m'envoyât au cinéma Le Styx, où l'on s'asseyait à l'époque dans des cercueils, voir L'enterré vivant, un film de Roger Corman tiré d'un conte d'Edgar Allan Poe. La découverte de la mort par le truchement de cette vision horrifique d'un homme qui hurle d'impuissance dans son cercueil devint une source capiteuse de cauchemars. Par la suite, je ne cessai de rechercher les attributs les plus spectaculaires de la mort [...] commencant à écrire, sous le pseudonyme d'Hector Lenoir, un conte qui racontait les affres d'un fantôme enchaîné dans les oubliettes du château des Hohenzollern.

Nous trouvons ici la confirmation de l'hypothèse voulant que Guibert ait subi, enfant, un traumatisme (l'opération des amygdales) qui lui a permis de conserver intacte et scellée dans la bouche une image idéale du père qu'il n'a pu nommer comme castrateur avant l'autobiographie. Ce père vivait dans la bouche fermée à autrui et à certains mots qui auraient dénoncé le crime paternel. De ce tombeau, nul ne parle, sauf le petit Guibert en

25 Guibert, Le protocole compassionnel, p. 68.

26 Ibid., p. 72. 
158

prenant bien soin de ne rien en dire. Hervé se complaît, enfant, dans les cercueils et aime les histoires d'enterrés vivants. Le sida et l'écriture de celui-ci réveille cette crypte dans la bouche, car pour se sauver du sida ou pour faire le deuil de lui-même, Guibert a besoin de sa bouche vide. Il a besoin de déterrer les morts. De là, l'enfermement dans la cave et l'ouverture de ce qui aurait pu constituer un tombeau pour un enterré vivant, de là, les désirs cannibaliques de chair non morte, vivante, puisque ces désirs tentent de liquider tout tombeau dans la bouche.

Qu'une certaine écriture soit liée au tombeau, il ne faut point en douter. Dans la cave, Guibert, emmuré, s'apprêtait à écrire et, enfant, il écrivit un conte sur un mort vivant, un fantôme oublié dans un château. Il est alors possible d'avancer que Guibert fut un sujet porteur de crypte au sens où Abraham et Torok l'entendent. Il a subi un refoulement conservateur qui l'empêche de faire le deuil d'un objet (les amygdales qui marquent le crime du père), objet qu'il garde intact dans un tombeau. L'autobiographie et surtout le sida vient déterrer les morts et demande au sujet l'ouverture de la crypte et la dénonciation du crime perpétré par le père. Rien d'étonnant à l'amour de la mort chez Guibert avant l'apparition du sida et cette attirance pour des sujets en deuil perpétuel. Lorsque Hervé veut se mettre à peindre, sa source d'inspiration est un tableau de Mancini, où l'on voit "un jeune garçon en costume de deuil ${ }^{27}$.

Cette passion pour le deuil vient inscrire ce qui du sujet cryptophore ne peut s'énoncer. L'attirance pour la mort dit: «il y ici un deuil qui insiste, un deuil dont on ne peut faire le deuil".

Ajoutons à cela un fait cocasse. Quand Guibert s'est fait fourrer la langue au fond de la gorge par la vieille putain fantasmatiquement porteuse et déclencheuse du sida, il dit être allé "se savonner les lèvres dans sa chambre d'hôtel de la rue EdgarAllan-Poe "28. C'est encore Edgar Allan Poe qui apparait dans le texte, tout comme il était apparu dans l'enfance, lors de la première rencontre avec la mort. Guibert avait vu à cette époque une adaptation d'un conte de Poe, appelée L'enterré vivant. Il est bel et bien question ici, au moment de l'apparition du sida, de crypte où gît un mort-vivant. Et le nom Edgar Allan Poe fonc-

27 Hervé Guibert, À l'ami qui ne m'a pas sauvé la vie, p. 72.

28 Ibid., p. 101 
tionne comme un passage entre la contamination par la putain et la crypte de l'enfance. C'est par ce signifiant que se fait le lien entre le sida et la crypte, et c'est grâce à ce signifiant que Guibert va réussir son élaboration d'une anti-incorporation de lui en tant qu'absence et mort prochaine. Ce moment d'ouverture de la crypte est extrêmement dangereux, car il se fait sous l'égide de la mort. Et le tombeau, la porte de la cave pourrait se refermer pour de bon. Mais Hervé Guibert va réouvrir les portes, va donner les clés de celles-ci (par exemple le nom Edgar Allan Poe est une clé) et va continuer à écrire non plus dans le tombeau mais dans un va-etvient incessant, dans une traversée perpétuelle du Styx, nom du cinéma où Guibert a vu L'enterré vivant.

Écrire, c'est ouvrir les tombeaux et commencer le deuil de soi. C'est à l'annonce de sa condamnation qu'Hervé Guibert va avoir une envie de vivre vorace et va abandonner sans cesse, pour sans cesse la reprendre, son attirance morbide. On peut lire à propos du sida ces mots terribles: "C'était une maladie qui donnait le temps de mourir et qui donnait à la mort le temps de vivre, le temps de découvrir le temps et de découvrir enfin la vie. " ${ }^{29}$ L'autobiographie aura été ce lieu privilégié où la mort a pu se dire et permettre encore un peu la vie.

Hervé Guibert est mort le 18 décembre 1991.

Ibid., p. 181. 